\title{
Erratum: Calculations of the atomic structure for the low-lying states of actinium [Phys. Rev. A 100, 022504 (2019)]
}

\author{
V. A. Dzuba $(0$, V. V. Flambaum, and B. M. Roberts (1)
}

(Received 17 April 2020; published 5 May 2020)

DOI: 10.1103/PhysRevA.101.059901

Our paper contained errors in calculated values of the electric dipole transition amplitudes and lifetimes of odd states. Energy levels were not affected. This was found by performing new calculations. Corrected values are presented in Tables I and II. Numeration of the states $\left(N_{o}\right.$ and $\left.N_{e}\right)$ is taken from our original paper, $N_{o}$ numerates odd states, and $N_{e}$ numerates even states. Transition amplitudes which differ from previous calculations more than two times are marked with asterisks. For other amplitudes, the agreement on the level of a few percent is for large amplitudes and up to 50\% is for small amplitudes. Table I presents all amplitudes needed for the calculation of lifetimes of the nine lowest odd states. Table III of our original paper presented only a fraction of these data. Odd states numbers 22 and 23 were missed in Table IV of the original paper.

TABLE I. Electric dipole transition amplitudes (reduced matrix elements in atomic units) among nine lowest odd states and all lower even states.

\begin{tabular}{|c|c|c|c|c|c|c|}
\hline \multicolumn{2}{|c|}{ Odd states } & \multicolumn{2}{|c|}{$N_{o}-N_{e}$} & \multicolumn{2}{|c|}{ Even states } & \multirow{2}{*}{$\frac{\text { Amplitude }}{1.7639}$} \\
\hline $7 s^{2} 7 p$ & ${ }^{2} P_{1 / 2}^{o}$ & 3 & 1 & $7 s^{2} 6 d$ & ${ }^{2} D_{3 / 2}$ & \\
\hline \multirow[t]{4}{*}{$7 s^{2} 7 p$} & ${ }^{2} P_{3 / 2}^{o}$ & 8 & 1 & $7 s^{2} 6 d$ & ${ }^{2} D_{3 / 2}$ & 0.5564 \\
\hline & & 8 & 2 & $7 s^{2} 6 d$ & ${ }^{2} D_{5 / 2}$ & $2.7251 *$ \\
\hline & & 8 & 4 & $7 s 6 d^{2}$ & ${ }^{4} F_{3 / 2}$ & 0.5812 \\
\hline & & 8 & 5 & $7 s 6 d^{2}$ & ${ }^{4} F_{5 / 2}$ & 0.7357 \\
\hline \multirow[t]{5}{*}{$7 s 7 p 6 d$} & ${ }^{4} F_{3 / 2}^{o}$ & 12 & 1 & $7 s^{2} 6 d$ & ${ }^{2} D_{3 / 2}$ & 1.4222 \\
\hline & & 12 & 2 & $7 s^{2} 6 d$ & ${ }^{2} D_{5 / 2}$ & $0.5030 *$ \\
\hline & & 12 & 4 & $7 s 6 d^{2}$ & ${ }^{4} F_{3 / 2}$ & 2.5923 \\
\hline & & 12 & 5 & $7 s 6 d^{2}$ & ${ }^{4} F_{5 / 2}$ & 0.4852 \\
\hline & & 12 & 10 & $7 s 6 d^{2}$ & ${ }^{4} P_{3 / 2}$ & 0.1032 \\
\hline \multirow[t]{9}{*}{$7 s 7 p 6 d$} & ${ }^{4} F_{5 / 2}^{o}$ & 15 & 1 & $7 s^{2} 6 d$ & ${ }^{2} D_{3 / 2}$ & 1.6269 \\
\hline & & 15 & 2 & $7 s^{2} 6 d$ & ${ }^{2} D_{5 / 2}$ & 1.4982 \\
\hline & & 15 & 4 & $7 s 6 d^{2}$ & ${ }^{4} F_{3 / 2}$ & 1.2340 \\
\hline & & 15 & 5 & $7 s 6 d^{2}$ & ${ }^{4} F_{5 / 2}$ & 2.9311 \\
\hline & & 15 & 6 & $7 s 6 d^{2}$ & ${ }^{4} F_{7 / 2}$ & 0.0685 \\
\hline & & 15 & 10 & $7 s 6 d^{2}$ & ${ }^{4} P_{3 / 2}$ & 0.0316 \\
\hline & & 15 & 11 & $7 s 6 d^{2}$ & ${ }^{4} D_{5 / 2}$ & 0.3969 \\
\hline & & 15 & 13 & $7 s 6 d^{2}$ & ${ }^{2} F_{5 / 2}$ & 0.2210 \\
\hline & & 15 & 14 & $7 s 6 d^{2}$ & ${ }^{2} D_{3 / 2}$ & 0.4773 \\
\hline \multirow[t]{6}{*}{$7 s 7 p 6 d$} & ${ }^{4} D_{1 / 2}^{o}$ & 18 & 1 & $7 s^{2} 6 d$ & ${ }^{2} D_{3 / 2}$ & 0.6240 \\
\hline & & 18 & 4 & $7 s 6 d^{2}$ & ${ }^{4} F_{3 / 2}$ & 3.1520 \\
\hline & & 18 & 9 & $7 s 6 d^{2}$ & ${ }^{4} P_{1 / 2}$ & 0.7208 \\
\hline & & 18 & 10 & $7 s 6 d^{2}$ & ${ }^{4} P_{3 / 2}$ & 0.1489 \\
\hline & & 18 & 14 & $7 s 6 d^{2}$ & ${ }^{2} D_{3 / 2}$ & 0.3500 \\
\hline & & 18 & 17 & $7 s 6 d^{2}$ & ${ }^{2} P_{1 / 2}$ & 0.2235 \\
\hline \multirow[t]{9}{*}{$7 s 7 p 6 d$} & ${ }^{4} D_{3 / 2}^{o}$ & 21 & 1 & $7 s^{2} 6 d$ & ${ }^{2} D_{3 / 2}$ & 0.7445 \\
\hline & & 21 & 2 & $7 s^{2} 6 d$ & ${ }^{2} D_{5 / 2}$ & 0.6392 \\
\hline & & 21 & 4 & $7 s 6 d^{2}$ & ${ }^{4} F_{3 / 2}$ & 1.5075 \\
\hline & & 21 & 5 & $7 s 6 d^{2}$ & ${ }^{4} F_{5 / 2}$ & 3.9777 \\
\hline & & 21 & 9 & $7 s 6 d^{2}$ & ${ }^{4} P_{1 / 2}$ & 1.0853 \\
\hline & & 21 & 11 & $7 s 6 d^{2}$ & ${ }^{4} D_{5 / 2}$ & 0.1932 \\
\hline & & 21 & 13 & $7 s 6 d^{2}$ & ${ }^{2} F_{5 / 2}$ & 0.4257 \\
\hline & & 21 & 14 & $7 s 6 d^{2}$ & ${ }^{2} D_{3 / 2}$ & 0.0793 \\
\hline & & 21 & 20 & $7 s 6 d^{2}$ & ${ }^{4} D_{5 / 2}$ & 0.2433 \\
\hline
\end{tabular}


TABLE I. (Continued.)

\begin{tabular}{|c|c|c|c|c|c|c|}
\hline \multicolumn{2}{|c|}{ Odd states } & \multicolumn{2}{|c|}{$N_{o}-N_{e}$} & \multicolumn{2}{|c|}{ Even states } & \multirow{2}{*}{$\frac{\text { Amplitude }}{1.1232}$} \\
\hline $7 s 7 p 6 d$ & ${ }^{4} F_{7 / 2}^{o}$ & 22 & 2 & $7 s^{2} 6 d$ & ${ }^{2} D_{5 / 2}$ & \\
\hline & & 22 & 5 & $7 s 6 d^{2}$ & ${ }^{4} F_{5 / 2}$ & 1.4187 \\
\hline & & 22 & 6 & $7 s 6 d^{2}$ & ${ }^{4} F_{7 / 2}$ & 3.8308 \\
\hline & & 22 & 7 & $7 s 6 d^{2}$ & ${ }^{4} F_{9 / 2}$ & 0.5212 \\
\hline & & 22 & 11 & $7 s 6 d^{2}$ & ${ }^{4} D_{5 / 2}$ & 0.4629 \\
\hline & & 22 & 13 & $7 s 6 d^{2}$ & ${ }^{2} F_{5 / 2}$ & 0.0015 \\
\hline & & 22 & 16 & $7 s 6 d^{2}$ & ${ }^{4} G_{7 / 2}$ & 0.0177 \\
\hline & & 22 & 19 & $7 s 6 d^{2}$ & ${ }^{2} G_{9 / 2}$ & 0.1330 \\
\hline & & 22 & 20 & $7 s 6 d^{2}$ & ${ }^{4} D_{5 / 2}$ & 0.2292 \\
\hline \multirow[t]{11}{*}{$7 s 7 p 6 d$} & ${ }^{2} D_{5 / 2}^{o}$ & 23 & 1 & $7 s^{2} 6 d$ & ${ }^{2} D_{3 / 2}$ & 1.2505 \\
\hline & & 23 & 2 & $7 s^{2} 6 d$ & ${ }^{2} D_{5 / 2}$ & 2.4129 \\
\hline & & 23 & 4 & $7 s 6 d^{2}$ & ${ }^{4} F_{3 / 2}$ & 0.4658 \\
\hline & & 23 & 5 & $7 s 6 d^{2}$ & ${ }^{4} F_{5 / 2}$ & 1.7723 \\
\hline & & 23 & 6 & $7 s 6 d^{2}$ & ${ }^{4} F_{7 / 2}$ & 1.7920 \\
\hline & & 23 & 10 & $7 s 6 d^{2}$ & ${ }^{4} P_{3 / 2}$ & 1.6800 \\
\hline & & 23 & 11 & $7 s 6 d^{2}$ & ${ }^{4} D_{5 / 2}$ & 2.072 \\
\hline & & 23 & 13 & $7 s 6 d^{2}$ & ${ }^{2} F_{5 / 2}$ & 3.051 \\
\hline & & 23 & 14 & $7 s 6 d^{2}$ & ${ }^{2} D_{3 / 2}$ & 0.5276 \\
\hline & & 22 & 16 & $7 s 6 d^{2}$ & ${ }^{4} G_{7 / 2}$ & 1.0293 \\
\hline & & 23 & 20 & $7 s 6 d^{2}$ & ${ }^{4} D_{5 / 2}$ & 0.2203 \\
\hline \multirow[t]{12}{*}{$7 s 7 p 6 d$} & ${ }^{4} D_{5 / 2}^{o}$ & 25 & 1 & $7 s^{2} 6 d$ & ${ }^{2} D_{3 / 2}$ & 0.4511 \\
\hline & & 25 & 2 & $7 s^{2} 6 d$ & ${ }^{2} D_{5 / 2}$ & 0.8195 \\
\hline & & 25 & 4 & $7 s 6 d^{2}$ & ${ }^{4} F_{3 / 2}$ & 0.0587 \\
\hline & & 25 & 5 & $7 s 6 d^{2}$ & ${ }^{4} F_{5 / 2}$ & 0.6827 \\
\hline & & 25 & 6 & $7 s 6 d^{2}$ & ${ }^{4} F_{7 / 2}$ & 4.8072 \\
\hline & & 25 & 10 & $7 s 6 d^{2}$ & ${ }^{4} P_{3 / 2}$ & 0.7703 \\
\hline & & 25 & 11 & $7 s 6 d^{2}$ & ${ }^{4} D_{5 / 2}$ & 0.9500 \\
\hline & & 25 & 13 & $7 s 6 d^{2}$ & ${ }^{2} F_{5 / 2}$ & 0.1039 \\
\hline & & 25 & 14 & $7 s 6 d^{2}$ & ${ }^{2} D_{3 / 2}$ & 0.6897 \\
\hline & & 25 & 16 & $7 s 6 d^{2}$ & ${ }^{4} G_{7 / 2}$ & 0.1281 \\
\hline & & 25 & 20 & $7 s 6 d^{2}$ & ${ }^{4} D_{5 / 2}$ & 0.2901 \\
\hline & & 25 & 24 & $7 s 6 d^{2}$ & ${ }^{4} G_{7 / 2}$ & 0.2807 \\
\hline
\end{tabular}

TABLE II. Calculated lifetimes of the nine low-lying odd states of Ac. Where available, experimental values of the transition frequency were used. The assigned errors include the uncertainty in the calculated frequencies and $E 1$ amplitudes.

\begin{tabular}{|c|c|c|c|c|c|}
\hline \multirow[b]{2}{*}{$N$} & & & \multicolumn{2}{|c|}{ Energy $\left(\mathrm{cm}^{-1}\right)$} & \multirow[b]{2}{*}{$\tau(\mathrm{ns})$} \\
\hline & \multicolumn{2}{|c|}{ State } & Expt. [1] & Calc. & \\
\hline 3 & $7 s^{2} 7 p$ & ${ }^{2} P_{1 / 2}^{o}$ & & 7565 & $733(70)$ \\
\hline 8 & $7 s^{2} 7 p$ & ${ }^{2} P_{3 / 2}^{o}$ & & 12345 & $238(20)$ \\
\hline 12 & $7 s 7 p 6 d$ & ${ }^{4} F_{3 / 2}^{o}$ & 13713 & 13958 & $317(30)$ \\
\hline 15 & $7 s 7 p 6 d$ & ${ }^{4} F_{5 / 2}^{o}$ & 14941 & 15141 & $196(20)$ \\
\hline 18 & $7 s 7 p 6 d$ & ${ }^{4} D_{1 / 2}^{o}$ & 17200 & 17049 & $139(14)$ \\
\hline 21 & $7 s 7 p 6 d$ & ${ }^{4} D_{3 / 2}^{o}$ & 17736 & 17612 & $142(14)$ \\
\hline 22 & $7 s 7 p 6 d$ & ${ }^{4} F_{7 / 2}^{o}$ & 17684 & 17715 & $385(40)$ \\
\hline 23 & $7 s 7 p 6 d$ & ${ }^{4} D_{5 / 2}^{o}$ & 17951 & 18108 & $80(8)$ \\
\hline 25 & $7 s 7 p 6 d$ & ${ }^{4} D_{5 / 2}^{o}$ & & 18747 & $157(16)$ \\
\hline
\end{tabular}

The authors are grateful to D. Budker and M. Safronova for pointing to inconsistencies in our previous results.

[1] A. Kramida, Y. Ralchenko, J. Reader, and The NIST ASD Team (2018), NIST Atomic Spectra Database (ver. 5.5.2) [https://physics.nist. gov/asd (2019)]. 